\title{
SCHWERPUNKTTHEMA „VFI-PREISTRÄGERINNEN 2014“
}

\section{Liebe Leserinnen und Leser,}

in der Geschichte des VFI-Förderungspreises, ${ }^{1}$ der seit 2006 für herausragende akademische Abschlussarbeiten aus dem deutschsprachigen Raum auf bestimmten Teilgebieten der Bibliotheks- und Informationswissenschaft vergeben wird, haben die Mitteilungen $\operatorname{der}$ VÖB stets eine Rolle gespielt - oder auch umgekehrt: in der jüngeren Geschichte der Mitteilungen $\operatorname{der} V O ̈ B$ hat der VFI-Förderungspreis eine nicht ganz unbedeutende Rolle gespielt. Einschliesslich der in der vorliegenden Ausgabe enthaltenen Beiträge wurden insgesamt sechzehn Artikel durch VFI-Preisträger/innen verfasst, wobei es sich stets um die durch den Preis initiierten Kurzfassungen der jeweils prämierten Magister-, Master- oder Doktorarbeiten handelte. Waren es zu Beginn meist Beiträge, die die betreffenden Preisträger/innen für die österreichische Leserschaft zu erstellen bereit waren, obwohl sie ihre mit dem Preis verbundene Publikationsverpflichtung bereits an anderer Stelle erfüllt hatten, so wandelte sich dies im Lauf der Jahre zusehends. Heute sind es nämlich in der Regel Artikel, die nicht indirekt, sondern direkt aus dieser Publikationsverpflichtung stammen. Zunehmend werden die Mitteilungen der VÖB als professionelles, referiertes Journal wahrgenommen, in dem zu veröffentlichen ähnlich attraktiv ist wie in anderen professionellen Zeitschriften des deutschen Sprachraumes.

Ende Januar dieses Jahres wurden die VFI-Preise für 2014 vergeben. Die aus sechs Personen bestehende Jury entschied sich für die Vergabe von zwei Hauptpreisen sowie von zwei Anerkennungspreisen. Folgende Arbeiten wurden prämiert:

\section{Hauptpreise:}

- Ingeborg Jäger-Dengler-Harles: Informationsvisualisierung und Retrieval im Fokus der Informationspraxis. Masterarbeit, Fachhochschule Köln, 2013. ${ }^{2}$ Diese Arbeit überzeugte durch eine gründliche Analyse des Einsatzes von Visualisierungsmethoden in der Informationspraxis, insbesondere im Information Retrieval, sowie einen fundierten Überblick über international realisierte Visualisierungsprojekte für Retrieval-Anwendungen und einen Ausblick auf künftige Entwicklungen. 
- Janine Lantzsch [nunmehr: Janine Saß]: Bestandsvisualisierung in Bibliotheken - Potenziale und Herausforderungen. Masterarbeit, Fachhochschule Potsdam, 2014. ${ }^{3}$ Diese thematisch verwandte Arbeit bietet eine gut strukturierte Analyse des Potentials von Bestandsvisualisierungen im Bereich Informationsrecherche und Angebotspräsentation in Bibliotheken sowie eine prägnante Beispielsammlung einschlägiger Anwendungen analoger und digitaler Art. Darüberhinaus wird eine eigene Konzeption für eine visuelle OPAC-Darstellung präsentiert.

\section{Anerkennungspreise:}

- Birte Lindstädt: Produkt- und Innovationsmanagement an Bibliotheken: Prozessfindung am Beispiel wissenschaftlicher Bibliotheken im deutschsprachigen Raum. Masterarbeit, Fachhochschule Köln, 2014. ${ }^{4}$ Die Arbeit beinhaltet eine Analyse und eine praxisrelevante Anleitung zur Umsetzung von Produkt- und Innovationsmanagement in Bibliotheken unter Berücksichtigung der Erfahrungen führender Bibliotheken aus dem deutschsprachigen Raum.

- Nora Schmidt: Semantisches Publizieren: Theoretische Grundlagen \& Anforderungen im interdisziplinären Wissenschaftsnetzwerk. Masterarbeit, Humboldt-Universität zu Berlin, 2014. ${ }^{5}$ Diese Arbeit stellt einen reflektierten Beitrag zur Theoriebildung im Bereich der Wissenschaftskommunikation unter besonderer Berücksichtigung der systemtheoretischen Sichtweise dar.

Die Redaktion der Mitteilungen war daran interessiert, die Kurzfassungen aller vier Kandidatinnen in einem Schwerpunktteil „VFI-Preisträgerinnen 2014“ zu publizieren. Dies wäre auch beinahe möglich gewesen, hätte nicht die vierte Preisträgerin den - vom VFI selbstverständlich akzeptierten - Wunsch geäussert, ihre Kurzversion in englischer Sprache in einem internationalen Journal zu veröffentlichen. ${ }^{6}$

Demzufolge bietet der folgende Schwerpunktteil die Aufsätze der ersten drei oben genannten Preisträgerinnen, die, wie ich meine, besonders interessante und aktuelle Themen behandeln. Danach folgt noch ein kurzer Artikel über die durch den VFI-Preis bisher initiierten Publikationen, wobei es vor allem um jene Beiträge geht, die nicht in unserer Zeitschrift erschienen sind - vielleicht kann dies den Appetit unserer Leserinnen und Leser wecken, auch in den betreffenden anderen Journalen nachzublättern. 
Ich wünsche Ihnen eine anregende Lektüre!

Beiträge und Feedback erbeten an: voeb-mitt@uibk.ac.at

Dr. Otto Oberhauser Editor des Schwerpunktthemas

E-Mail: oberh@web.de

1 vgl.: http://www.vfi-online.org/ [alle zitierten Webadressen wurden am 27.10.2015 überprüft]

2 Volltext: http://publiscologne.th-koeln.de/frontdoor/index/index/docld/334

3 Volltext: https://opus4.kobv.de/opus4-fhpotsdam/frontdoor/index/index/docld/776

4 Volltext: http://publiscologne.th-koeln.de/frontdoor/index/index/docld/644

5 Volltext: http://edoc.hu-berlin.de/series/berliner-handreichungen/2014-375/ PDF/375.pdf

6 Da sich dieser Beitrag noch im Reviewprozess befindet, kann hier noch kein bibliographischer Hinweis gegeben werden.

(c) (7)

Dieses Werk ist lizenziert unter einer

Creative-Commons-Lizenz Namensnennung 4.0 International 